\title{
An Axiomatic Presentation of the Nonstandard Methods in Mathematics
}

\author{
Mauro Di Nasso
}

\begin{abstract}
A nonstandard set theory ${ }^{*} \mathrm{ZFC}$ is proposed that axiomatizes the nonstandard embedding $*$. Besides the usual principles of nonstandard analysis, all axioms of ZFC except regularity are assumed. A strong form of saturation is also postulated. ${ }^{*} \mathrm{ZFC}$ is a conservative extension of $\mathrm{ZFC}$.
\end{abstract}

\section{Introduction.}

For practitioners, the most popular foundational approach to nonstandard methods is the so-called superstructure approach, introduced by A. Robinson and E. Zakon in [RZ]. Roughly speaking, it consists of two superstructures $V(X)$ and $V(Y)$, namely the standard model and the nonstandard model, and of a mapping $*: V(X) \rightarrow V(Y)$, namely the nonstandard embedding, which provides a transfer principle for elementary properties. The $\kappa$-saturation property is also usually assumed, for a suitable cardinal $\kappa .^{1}$ Though well-suited for applications, such an approach reveals limitations from a foundational point of view. Superstructures consist of sets of finite rank in the cumulative hierarchy, thus modeling only a fragment of set theory, and different superstructures are needed to treat different problems. Another criticism is that, in principle, nonstandard methods are not concerned with superstructures. Also, it seems esthetically desirable to include the nonstandard techniques within a unified axiomatic system. Since the seventies, the search for a general framework for nonstandard methods led to the formulation of several axiomatic approaches. ${ }^{2}$ Those studies revealed serious foundational difficulties. Most notably, the core problem that every nonstandard theory has to deal with is Hrbăček paradox [HR], namely the inconsistency of the usual principles of nonstandard analysis and set theory, in the presence of unlimited levels of saturation. In this paper we show that the above paradox can be overcome because universes of full nonstandard set theory can be constructed which have, in some precise sense, the "feel" of unlimited saturation. The axiomatic theory * ZFC presented in this paper is an extension of the traditional foundational framework of mathematics, namely ZFC, where

\footnotetext{
${ }^{1}$ For an introduction to nonstandard analysis and the foundations of the superstructure approach, we refer the reader to $[\mathrm{HL}]$ and $[\mathrm{CK}] \S 4.4$, respectively.

${ }^{2} \mathrm{~A}$ survey of nonstandard set theories is given in [D3].
} 
only the regularity axiom is dropped. Nonstandard arguments can be embedded in ${ }^{*} \mathrm{ZFC}$ in a fashion that is consistent with the superstructure approach. Thus, the superstructure practitioner willing to adopt ${ }^{*} \mathrm{ZFC}$ as an axiomatic system, needs to change nothing with respect to his/her familiar definitions and notation, and he/she can carry out all usual nonstandard proofs without change. Other features of ${ }^{*} \mathrm{ZFC}$ are that all objects of ordinary mathematics can be considered as standard sets, and that external sets are available without restrictions, because all constructions of ordinary mathematics are allowed. Moreover, a strong form of saturation is assumed which, in most cases occurring in practice, is virtually equivalent to unlimited saturation. As for its strength as a formal system, ${ }^{*} \mathrm{ZFC}$ is a conservative extension of ZFC. This is a straight consequence of a more general result (Theorem 3.3) stating that every model of ZFC is elementarily embedded into the wellfounded part of some model of ${ }^{*}$ ZFC. The theory ${ }^{*} \mathrm{ZFC}$ was first presented at the International Congress on Nonstandard Analysis and its Applications, held in Edinburgh, August 1996. A previous version appeared in [D2].

Throughout the paper, we will work in Zermelo-Fraenkel set theory with choice, but we will not assume regularity except where explicitly noted. We shall use freely standard facts and notation of set theory and model theory, the default references being $[\mathrm{KU}]$ and $[\mathrm{CK}]$, respectively. In a nonwellfounded context, an ordinal is defined as a transitive set which well-ordered by $\in$. A cardinal is an initial ordinal. We informally use classes to denote extensions of formulas in the style of [Ku], and use boldface letters to denote them. A proper class is a class which is not a set. $\mathbf{V}$ will denote the universal class of all sets; WF the class of wellfounded sets; and $\mathbf{O N}$ the class of ordinals.

\section{$\S 1$. The theory ${ }^{*}$ ZFC.}

The theory ${ }^{*} \mathrm{ZFC}$ is formulated in the first-order language consisting of a symbol $\in$ for the membership relation and of a symbol $*$ for the nonstandard embedding. Its axioms are the following six groups of sentences.

\section{1. $Z F C^{-}$}

All axioms of Zermelo-Fraenkel set theory with choice are assumed, with the only exception of regularity. The separation and replacement schemata are also assumed for formulas cointaining the $*$ symbol.

Thus all arguments of ordinary mathematics can be formalized within *ZFC. We remark that the axiom of regularity cannot be assumed if we want a (nontrivial) nonstandard extension for each infinite set. For instance, as any nonstandard model of the natural number system is not wellordered, the nonstandard extension ${ }^{*} \omega$ of the Von Neumann natural numbers is necessarily nonwellfounded. On the other hand, regularity is never used in the practice of mathematics, with the only exception of some results in set theory and general 
topology. ${ }^{3}$

Definition. The class $\mathbf{S}$ of standard sets is the class WF of wellfounded sets.

In particular, elements of standard sets are standard sets and the collection $\mathbf{S}$ of standard sets is a model of ZFC. In the "standard" set theory, every set is wellfounded. Thus it seems appropriate to consider $\mathbf{S}$ as the universe of "standard" mathematics. In the following, " $x \in \mathbf{S}$ " will be a short-hand for the $\in$-formula that says: " $x$ is a wellfounded set", that is

$\exists t$ transitive s.t. $x \subseteq t \wedge \forall y \in t(y \neq \emptyset \rightarrow \exists z \in y z \cap y=\emptyset)$

2. * gives a mapping with domain $\mathbf{S}$.

$$
\{\forall x \forall y \forall z[*(x, y) \wedge *(x, z) \rightarrow(y=z \wedge x \in \mathbf{S})]\} \wedge(\forall x \in \mathbf{S} \exists y *(x, y))
$$

As $*$ is not defined for all sets, we had to formally consider it as a binary relation symbol. However, in the following we will abuse notation and directly use $*$ as a function. " The above axiom states that each object $A$ of "standard" mathematics, has its nonstandard extension * $A$. Exactly as in the superstructure approach, we give the

Definition. The class of internal sets is the collection $\mathbf{I}=\left\{a: a \in{ }^{*} b\right.$ for some $b \in \mathbf{S}\}$. A set $x \notin \mathbf{I}$ is called external.

The next axiom postulates a convenient property for internal sets which is always assumed in the literature.

3. $\mathbf{I}$ is a transitive class, i.e. elements of internal sets are internal.

$$
\forall x \forall y(x \in y \wedge y \in \mathbf{I}) \rightarrow x \in \mathbf{I}
$$

4. Transfer principle schema.

For every $\in$-formula $\varphi\left(x_{1}, \ldots, x_{n}\right)$, the following is an axiom: ${ }^{5}$

$$
\forall a_{1}, \ldots, a_{n} \in \mathbf{S} \varphi^{S}\left(a_{1}, \ldots, a_{n}\right) \leftrightarrow \varphi^{I}\left({ }^{*} a_{1}, \ldots,{ }^{*} a_{n}\right)
$$

\footnotetext{
${ }^{3}$ Typical examples of such results are theorems in set theory concerning the structure of the universal class, namely the Mostowski collapse theorem, the Cumulative hierarchy theorem and the $\in$-induction theorem. Notice that in a nonwellfounded context, every result which is essentially grounded on the axiom of regularity can be directly reformulated as a property relative to the class of wellfounded sets. For instance, Mostowski theorem can be restated as follows: "Any extensional wellfounded binary structure is isomorphic to a transitive wellfounded set", etc.

${ }^{4}$ Precisely, we shall write " $y={ }^{*} x "$ instead of " $\forall z *(x, z) \rightarrow z=y$ ", and " $y \in{ }^{*} x "$ instead of " $\forall z *(x, z) \rightarrow y \in z$ ". When writing * $x$, we implicitly assume that $x \in \mathbf{S}$.

${ }^{5}$ Recall the notion of relativized formula. If $\mathbf{C}=\{x: \sigma(x)\}$ is a class and $\varphi$ is a formula, the relativization $\varphi^{C}$ is the formula obtained from $\varphi$ by replacing each quantifier $\forall x$ and $\exists x$ with its restricted form $\forall x \in \mathbf{C}$ (i.e. $\forall x \sigma(x) \rightarrow \ldots$ ) and $\exists x \in \mathbf{C}$ (i.e. $\exists x \sigma(x) \wedge \cdots$ ), respectively.
} 
In other words, a first-order property (in the language of set theory) holds in the standard universe $\mathbf{S}$ about standard objects $a_{1}, \ldots, a_{n}$ if and only if the same property holds in the internal universe $\mathbf{I}$ about the corresponding nonstandard extensions ${ }^{*} a_{1}, \ldots,{ }^{*} a_{n}$. In a model-theoretic language, the transfer principle postulates that the nonstandard extension map $*:\langle\mathbf{S}, \in\rangle \rightarrow\langle\mathbf{I}, \in\rangle$ is an elementary embedding. We remark that the above transfer principle schema can be equivalently reformulated as a single axiom. Precisely, by assuming 1 and $\mathbf{2}$, the following is proved: ${ }^{6}$

- 3 and $\mathbf{4}$ hold if and only if all Gödel's operations are preserved under *. ${ }^{7}$

We now turn to an axiom on the $\in$-structure of the universal class. It is a weakened form of regularity that can be retained in our nonwellfounded context. Although it has relevance from a (nonstandard) set-theoretic point of view, we remark that it has no effect on the practice of nonstandard analysis.

5. Weak Regularity.

Every set is wellfounded over the class of internal sets.

$$
\forall x \neq \emptyset \exists y \in x x \cap y \subseteq \mathbf{I}
$$

It is easily proved (by assuming the previous axioms) that weak regularity is equivalent to the non-existence of $\in$-descending chains $a_{o} \ni a_{1} \ni \ldots \ni a_{n} \ni \ldots$ where all $a_{n}$ 's are external sets.

A fundamental tool in nonstandard analysis is the following $\kappa$-saturation property, where $\kappa$ is a given cardinal.

"If a (nonempty) family of internal sets has cardinality less than $\kappa$ and it is closed under intersections, then it has nonempty intersection"

$$
[\emptyset \neq \mathcal{F} \subseteq \mathbf{I} \wedge|\mathcal{F}|<\kappa \wedge \forall A, B \in \mathcal{F}(\emptyset \neq A \cap B \in \mathcal{F})] \rightarrow \bigcap \mathcal{F} \neq \emptyset
$$

6. Saturation Schema.

If a cardinal $\kappa$ is defined by an $\in$-formula in $\mathbf{S}$, then the $\kappa$-saturation property holds. More formally, for every $\in$-formula $\varphi(x)$ having exactly one free variable, the following is assumed.

$$
\begin{gathered}
{\left[\forall x, y \in \mathbf{S} \varphi^{S}(x) \wedge \varphi^{S}(y) \rightarrow(x=y \wedge \text { " } x \text { is a cardinal" })\right]} \\
\Downarrow \\
\forall \kappa\left[\varphi^{S}(\kappa) \rightarrow \text { " } \kappa \text {-saturation property" }\right]
\end{gathered}
$$

\footnotetext{
${ }^{6}$ See for instance [D4].

${ }^{7}$ A complete list of Gödel's operations can be found in [JE] $\S 11$.
} 
Though formally no constant symbol is allowed in the language of set theory, nevertheless in practice certain cardinals are used as constants (e.g. 17, $\omega, \aleph_{13}$, Beth $_{\omega}$, the first inaccessible cardinal, the 7 th measurable cardinal, the least cardinal such that a certain property fails, etc.) This can be done because such cardinals are uniquely defined by a formula and it is possible to consider them as "definable" constants, irrespective whether their existence is provable or not. Roughly speaking, this means that every cardinal that can be explicitly mentioned is "definable". E.g. the following is an axiom of *ZFC: "If the first inaccessible cardinal $\nu$ exists, then the $\nu$-saturation property holds."

$\S 2$. Working in $* Z F C$.

The next result shows that, in typical situations occurring in practice, saturation schema 6 provides the full strength of saturation, without restrictions on cardinalities. Let $\varphi(x)$ be a given $\in$-formula having exactly one free variable.

Theorem 2.1 ( $\left.{ }^{*} \mathrm{ZFC}\right)$

Assume the following: "For every cardinal $\kappa, \kappa$-saturation $\Rightarrow \varphi^{S}(x)$ for all standard $x$ of cardinality less that $\kappa "$. Then $\varphi^{S}(x)$ holds for all standard $x$.

Proof. By contradiction, assume that $\varphi^{S}(x)$ fails for some standard $x$. Let $\nu$ be the least cardinality of a counter-example $a$. Notice that $\nu$ is defined by an $\in$-formula in $\mathbf{S}$. Hence, the $\nu^{+}$-saturation property holds, and by the hypothesis $\varphi^{S}(x)$ holds for all standard $x$ with $|x| \leq \nu$. A contradiction with $\neg \varphi^{S}(a)$.

Suppose that for any $\kappa$, we can prove that a certain standard property is true for all standard sets with cardinality less than $\kappa$, provided $\kappa$-saturation is assumed. Then, by the previous theorem, that property is actually proved for all standard sets. A typical example that shows how theorem 2.1 can be used in practice, is the following. Recall the nonstandard characterization of compactness in topology.

(•) Assume $\kappa$-saturation. If $X$ is a (standard) topological Hausdorff space with $|X|<\kappa$, then $X$ is compact if and only if for every $\xi \in{ }^{*} X$, there is some $x \in X$ with $\xi \approx^{*} x .^{8}$

Making use of $(\bullet)$, a nice and short nonstandard proof of Tychonoff theorem is obtained. Precisely, the following is proved

For all families $\left\{X_{i}: i \in I\right\}$ of compact topological Hausdorff spaces where $\left|X_{i}\right|<\kappa$ for all $i \in I$ and $|I|<\kappa$, the topological product space $\prod_{i} X_{i}$ is compact.

By theorem 2.1, the above result actually proves Tychonoff theorem for all standard topological spaces, without restrictions on cardinalities.

\footnotetext{
${ }^{8}$ We used notation $\xi \approx{ }^{*} x$ to mean that $\xi$ belongs to the monad of $x$, that is $\xi \in{ }^{*} A$ for each (standard) neighborhood $A$ of $x$.
} 
A consequence of the axiom of choice is that every set is in bijection with some ordinal. In particular, the following holds.

Proposition $2.2\left({ }^{*} \mathrm{ZFC}\right)$

Every set is in bijection with some standard set.

As a consequence, one can apply the map $*$ to any (possibly external) mathematical structure, simply by considering an isomorphic standard copy of it. For example, if $\mathcal{L}$ is a Loeb measure space, one can take a standard isomorphic copy $\mathcal{L}^{\prime} \cong \mathcal{L}$, consider its nonstandard extension ${ }^{*} \mathcal{L}^{\prime}$, and then apply the nonstandard methods to it. ${ }^{9}$ The next theorem gives some information about the structure of the universe of *ZFC.

Theorem $2.3\left({ }^{*} \mathrm{ZFC}\right)$

(i) The fixed points of $*$ are precisely the hereditarily finite sets. That is, $a=$ ${ }^{*} a \Leftrightarrow a \in V_{\omega} .10$

(ii) The universal class of all sets is given by the union $\mathbf{V}=\bigcup_{\alpha \in O N} V_{\alpha}\left({ }^{*} V_{\alpha}\right)$.

(iii) For all standard sets $A$ and for all ordinals $\alpha, V_{\alpha}\left({ }^{*} A\right) \cap \mathbf{I} \subseteq{ }^{*} V_{\alpha}(A)$. In particular, for all ordinals $\alpha$ and $\beta, V_{\beta}\left({ }^{*} V_{\alpha}\right) \cap \mathbf{I} \subseteq{ }^{*} V_{\alpha+\beta}$.

(iv) For every ordinal $\alpha$, there is a limit ultrapower $V_{\alpha}^{I} \mid F$ and an isomorphism $\pi_{\alpha}: V_{\alpha}{ }_{D}^{I} \mid F \cong\left\langle{ }^{*} V_{\alpha}, \in\right\rangle$ such that the following diagram commutes: ${ }^{11}$

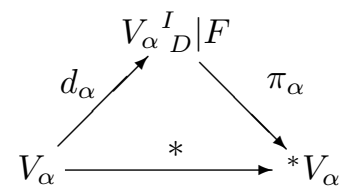

Proof. (i) $\Leftarrow$ is easily proved by induction on the finite $\operatorname{rank} \rho(a)=\min \{k: a \subseteq$ $\left.V_{k}\right\}$. Vice versa, it is enough to prove that for every standard $B \notin V_{\omega},{ }^{*} B$ is not wellfounded. To this end, transfer the property " $\forall n \in \omega \exists x \in B \quad x \notin V_{n}$ " and get " $\forall \xi \in{ }^{*} \omega \exists x \in{ }^{*} B x \notin V_{\xi}$ " (here we are considering the standard mapping $n \mapsto V_{n}$ for $n \in \omega$ and its nonstandard extension $\xi \mapsto V_{\xi}$ for $\left.\xi \in{ }^{*} \omega\right)$. Take an infinite $\xi$ (i.e. $\xi>n$ for all $n \in \omega$ ) and $b_{1} \in{ }^{*} B$ with $b_{1} \notin V_{\xi}$. Then $b_{1} \nsubseteq V_{\xi-1}$ and there is an element $b_{2} \in b_{1}$ with $b_{2} \notin V_{\xi-1}$. By iterating, we get an $\in$-descending chain ${ }^{*} B \ni b_{1} \ni b_{2} \ni \ldots$

(ii) The following property holds:

"For any set $x$, if $x \subseteq \bigcup_{\alpha \in O N} V_{\alpha}\left({ }^{*} V_{\alpha}\right)$, then $x \in V_{\alpha}\left({ }^{*} V_{\alpha}\right)$ for some $\alpha$ "

In fact, for each $\xi \in x$, let $\alpha_{\xi} \doteq \operatorname{Min}\left\{\alpha: \xi \in V_{\alpha}\left({ }^{*} V_{\alpha}\right)\right\}$. By replacement, the collection $\left\{\alpha_{\xi}: \xi \in x\right\}$ is a set and we can consider its supremum $\lambda$. Thus

\footnotetext{
${ }^{9}$ Actually, with some limitation in the use of saturation. In fact, the cardinality of such a set $\mathcal{L}^{\prime}$ is larger than any $\in$-definable cardinal.

${ }^{10}$ Recall the cumulative hierarchy over a given set $X . V_{0}(X)=X ; V_{\alpha+1}(X)=V_{\alpha}(X) \cup$ $\mathcal{P}\left(V_{\alpha}(X)\right) ; V_{\beta}(X)=\bigcup_{\alpha<\beta} V_{\alpha}(X)$ if $\beta$ is limit. We simply write $V_{\alpha}$ to mean $V_{\alpha}(\emptyset)$.

${ }^{11}$ Limit ultrapowers are a generalization of the ultrapowers. Definition and basic results can be found in $[\mathrm{CK}] \S 6.4 . d_{\alpha}$ denotes the canonical diagonal embedding.
} 
$x \subseteq V_{\lambda}\left({ }^{*} V_{\lambda}\right)$, hence $x \in V_{\lambda+1}\left({ }^{*} V_{\lambda+1}\right)$. Now, assume by contradiction that there is a set $a$ with $a \notin V_{\alpha}\left({ }^{*} V_{\alpha}\right)$ for every $\alpha$. By the above property $a \nsubseteq \bigcup_{\alpha} V_{\alpha}\left({ }^{*} V_{\alpha}\right)$ for all $\alpha$, so there is an element $a_{1} \in a$ with $a_{1} \notin V_{\alpha}\left({ }^{*} V_{\alpha}\right)$ for all $\alpha$. By iterating, we obtain an $\in$-descending chain of external elements $a \ni a_{1} \ni a_{2} \ni \ldots$ against weak regularity.

(iii) Proceeding by transfinite induction on $\alpha$, the only nontrivial case is when $\alpha=\beta+1$ is a successor. If $\xi \in V_{\beta+1}\left({ }^{*} A\right) \backslash V_{\beta}\left({ }^{*} A\right)=\mathcal{P}\left(V_{\beta}\left({ }^{*} A\right)\right)$ is internal, then by transitivity of $\mathbf{I}$ and by the inductive hypothesis, $\xi \subseteq{ }^{*} V_{\beta}(A)$. By transferring the standard $\in$-property, we get:

$$
\forall x \in \mathbf{I}\left[\left(\forall y \in x y \in{ }^{*} V_{\beta}(A)\right) \rightarrow x \in{ }^{*} V_{\beta+1}(A)\right]
$$

In particular, $\xi \in{ }^{*} V_{\beta+1}(A)$.

(iv) The restriction $*:\left\langle V_{\alpha}, \in\right\rangle \rightarrow\left\langle{ }^{*} V_{\alpha}, \in\right\rangle$ is a complete embedding, i.e. an elementary embedding with respect to the complete language containing one symbol for each constant, function and relation over $V_{\alpha}$. This fact straightforwardly follows from the transfer principle schema. Then apply Keisler's characterization theorem on limit ultrapowers (see [CK] Theorem 6.4.10).

Now, let $\sigma(x)$ be an $\in$-*-formula.

Theorem $2.4 \in$-Induction over I ( $\left.{ }^{*} \mathrm{ZFC}\right)$

Suppose that $\forall x \in \mathbf{I} \sigma(x)$ and suppose that, for every set $A$, the following holds: $(\forall a \in A \sigma(a)) \rightarrow \sigma(A)$. Then $\forall x \sigma(x)$.

Proof. By contradiction, assume $\neg \sigma(A)$ for some set $A$. Then there must be some $a_{1} \in A$ with $\neg \sigma\left(a_{1}\right)$. Notice that $\neg \sigma\left(a_{1}\right) \Rightarrow a_{1} \notin \mathbf{I}$. Again, there must be an element $a_{2} \in a_{1}$ with $\neg \sigma\left(a_{2}\right)$ and $a_{2} \notin \mathbf{I}$. By iterating we get an $\in$-descending chain $A \ni a_{1} \ni a_{2} \ni \ldots$ of external elements, against weak regularity.

Let $W_{\alpha}=V_{\alpha}\left({ }^{*} V_{\alpha}\right)$ if $\alpha$ is a successor ordinal, and let $W_{\alpha} \doteq \bigcup_{\beta<\alpha} W_{\beta}$ if $\alpha$ is limit. ${ }^{12}$ Then for any given $\in$-*-sentence $\sigma$, the following holds.

Theorem 2.5 Reflection Principle ( $\left.{ }^{*} \mathrm{ZFC}\right)$

For every ordinal $\alpha$, there is $\beta>\alpha$ with $\sigma \leftrightarrow \sigma^{W_{\beta}}$.

Proof. Recall that $\mathbf{V}=\bigcup_{\alpha \in O N} W_{\alpha}$. Since $\alpha \leq \beta \Rightarrow W_{\alpha} \subseteq W_{\beta}$ and $W_{\alpha}=$ $\bigcup_{\beta<\alpha} W_{\beta}$ if $\alpha$ is limit, one can carry out within ${ }^{*} \mathrm{ZFC}$ the same proof of the Reflection Principle as done within ZFC (see for instance [KU] Ch.IV §7.).

We now prove the fundamental.

Theorem 2.6 Standardization Property (*ZFC)

For every set $A$, the following collections are sets: (i) ${ }^{o} A \doteq\left\{b \in \mathbf{S}:{ }^{*} b \in A\right\} \in \mathbf{S}$; (ii) ${ }^{\sigma} A \doteq\left\{a \in A: a={ }^{*} b\right.$ for some $\left.b \in \mathbf{S}\right\}$.

Proof. By theorem 2.3, $A \cap \mathbf{I} \subseteq V_{\alpha}\left({ }^{*} V_{\alpha}\right) \cap \mathbf{I} \subseteq{ }^{*} V_{\alpha+\alpha}$ for some $\alpha$. By the separation axiom, it is straighforwardly seen that the collection ${ }^{o} A=\{b \in$

${ }^{12}$ Notice that $W_{\alpha}$ is a proper subset of $V_{\alpha}\left({ }^{*} V_{\alpha}\right)$ for all limit $\alpha$. 
$\left.V_{\alpha+\alpha}:{ }^{*} b \in A\right\}$ is a set. Also (ii) directly follows from the separation axiom.

The standardization property states that the preimage of any set under the function $*$ is a set. This seems to be a very natural property to have, in that it allows the use of the nonstandard embedding when defining sets. Although it is trivially satisfied in the superstructure approach, we remark that in most of the axiomatic approaches appeared in the literature, standardization is either added as an axiom (e.g. Hrbacek's NS ${ }_{1}$, Nelson's IST, Fletcher's SNST) or it is false in its general form (e.g. Kawai's NST). ${ }^{13}$

The axiom of regularity implies the existence of a wellordered valued rank function $\rho: \mathbf{V} \rightarrow \mathbf{O N}$ where $\rho(a)=\sup \left\{\rho\left(a^{\prime}\right)+1: a^{\prime} \in a\right\}$ if $a \neq \emptyset$ and $\rho(\emptyset)=0$. Although nonwellfounded, the universe of $*$ ZFC retains a "pseudorank" function. Precisely, the following holds.

Theorem 2.7 ( $\left.{ }^{*} \mathrm{ZFC}\right)$

There is a linearly ordered class $\lambda$ and a rank function $\mathbf{R}: \mathbf{V} \rightarrow \lambda$ that satisfies: (i) $\mathbf{R}(x)=\sup \left\{\mathbf{R}\left(x^{\prime}\right)+1: x^{\prime} \in x\right\}$ if $x \neq \emptyset$ and $\mathbf{R}(\emptyset)=0 .{ }^{14}$

(ii) $\mathbf{R}\left({ }^{*} a\right)={ }^{*} \rho(a)$ for every $a \in \mathbf{S}$.

(iii) $\mathbf{R}(\xi)=\xi$ for all internal-ordinals $\xi \in{ }^{*} \mathbf{O N}=\bigcup_{\alpha \in O N}{ }^{*} \alpha$.

Proof. It is a modification of arguments in the proof of the Representation Theorem of [D1]. Thus we only give a sketch and refer the reader to that paper for more details. The starting point is the familiar von Neumann rank $\rho: \mathbf{S} \rightarrow \mathbf{O N}$ defined on the class of wellfounded sets. By transfer we get a rank function for internal sets $\mathcal{R}: \mathbf{I} \rightarrow{ }^{*} \mathbf{O N}$ where $\mathcal{R}(x)=\sup \left\{\mathcal{R}\left(x^{\prime}\right)+1: x^{\prime} \in x\right\}$ if $x \neq \emptyset$ and $\mathcal{R}(\emptyset)=0$. Precisely, $\mathcal{R}$ is defined as the union $\bigcup_{\alpha}{ }^{*} \rho_{\alpha}$ where $\rho_{\alpha}: V_{\alpha} \rightarrow \alpha$ is the restriction of $\rho$ to the first $\alpha$ levels in the hierarchy. Now, let the ordinal $\alpha$ be given. Take $\left\langle\lambda_{\alpha}^{\prime},<\right\rangle$ to be the Dedekind order-completion of the linearly ordered set $\left\langle{ }^{*} \alpha, \in\right\rangle$. Then replace every new element $x \in \lambda_{\alpha}^{\prime} \backslash^{*} \alpha$ with a copy $\mathbf{O N}_{x}=\left\{\beta_{x}: \beta \in \mathbf{O N}\right\}$ of the ordinals, and identify $0_{x}$ with $x$. For each $a \in \mathbf{W F}\left({ }^{*} V_{\alpha}\right) \doteq \bigcup_{\beta \in O N} V_{\beta}\left({ }^{*} V_{\alpha}\right)$, define $R_{\alpha}(a)=\mathcal{R}(a)$ if $a \in{ }^{*} V_{\alpha}$ and $R_{\alpha}(a)=\sup \left\{R_{\alpha}\left(a^{\prime}\right)+1: a^{\prime} \in a\right\}$ otherwise. Making use of (iii) in theorem 2.3, it is proved by transfinite induction that $a \in \mathbf{W F}\left({ }^{*} V_{\alpha}\right) \cap \mathbf{I} \Rightarrow R_{\alpha}(a)=\mathcal{R}(a)$. Thus $R_{\alpha}$ is compatible with $\mathcal{R}$. Repeat this construction of $\lambda_{\alpha}$ and $R_{\alpha}$ for all ordinals $\alpha$. Notice that, without loss of generality, we can assume $\lambda_{\beta} \subseteq \lambda_{\alpha}$ when $\beta \leq \alpha$. Since $\left\{R_{\alpha}: \alpha \in \mathbf{O N}\right\}$ is a family of pairwise compatible functions, by taking the unions $\mathbf{R}=\bigcup_{\alpha} R_{\alpha}$ and $\lambda=\bigcup_{\alpha} \lambda_{\alpha}$, the universal rank function $\mathbf{R}: \mathbf{V} \rightarrow \lambda$ is obtained. $\mathbf{R}$ satisfies (i) by definition of the $R_{\alpha}$ 's. To prove (ii), notice that if $a \in V_{\alpha}$ for some $\alpha$, then $\mathbf{R}\left({ }^{*} a\right)=\mathcal{R}\left({ }^{*} a\right)={ }^{*} \rho_{\alpha}\left({ }^{*} a\right)=$ ${ }^{*}\left[\rho_{\alpha}(a)\right]={ }^{*}[\rho(a)]$. As for (iii), if $\xi \in{ }^{*} \alpha$ for some ordinal $\alpha$, apply transfer to

\footnotetext{
${ }^{13}$ See [D3].

${ }^{14}$ It is implicitly assumed that $\lambda$ has a least element 0 , that every $\xi \in \lambda$ has immediate successor $\xi+1$ and that every collection of the form $\left\{\mathbf{R}\left(x^{\prime}\right)+1: x^{\prime} \in x\right\}$ has a least upper bound in $\lambda$.
} 
the standard property " $\forall x \in \alpha \rho_{\alpha}(x)=x$ " and get ${ }^{*} \rho_{\alpha}(\xi)=\xi$, hence $\mathbf{R}(\xi)=\xi$.

\section{§3. Justifying $*$ ZFC.}

Denote by $L_{*}=\{\in, *\}$ the language of *ZFC. In the following we will argue in ZFC (including regularity).

Definition. An inner model $\mathcal{U}$ for the language $L_{*}$ is determined by a formula $u(x)$, which defines the universe $\mathbf{U}=\{a: u(a)\}$; by a formula $E(x, y)$ defining the interpretation of the membership relation symbol; and by a formula $\otimes(x, y)$ defining the interpretation of the $*$ symbol.

For convenience, we will abuse notation and write $a \mathbf{E} b$ instead of $E(a, b)$ and denote the inner model $\mathcal{U}=\langle\mathbf{U}, \mathbf{E}, \otimes\rangle$ as a triplet. Notice that in general $\mathbf{U}, \mathbf{E}$ and $\otimes$ are proper classes. The satisfaction relation is straightforwardly defined by induction on the complexity of formulas. ${ }^{15}$ Starting from formulas $u(x, p), e(x, y, p), \otimes(x, y, p)$, one can similarly consider the inner models $\mathcal{U}_{p}=$ $\left\langle\mathbf{U}_{p}, \mathbf{E}_{p}, \otimes_{p}\right\rangle$ where $p$ is a parameter. By functional correspondance we mean a class which is a function, i.e. a class of ordered pairs $\Theta=\{\langle x, y\rangle: \vartheta(x, y)\}$, where $\vartheta(x, y) \wedge \vartheta\left(x, y^{\prime}\right) \rightarrow y=y^{\prime}$.

Definition. A functional correspondance $\Theta$ is an $\in$-*-isomorphism between the inner models $\mathcal{U}=\langle\mathbf{U}, \mathbf{E}, \otimes\rangle$ and $\mathcal{U}^{\prime}=\left\langle\mathbf{U}^{\prime}, \mathbf{E}^{\prime}, \otimes^{\prime}\right\rangle$ if:

(i) $\Theta: \mathbf{U} \rightarrow \mathbf{U}^{\prime}$ is a bijection;

(ii) $x \mathbf{E} y \Leftrightarrow \Theta(x) \mathbf{E}^{\prime} \Theta(y)$

(iii) $\otimes(x, y) \Leftrightarrow \otimes^{\prime}(\Theta(x), \Theta(y))$.

In case only (i) and (ii) are satisfied, we say that $\Theta$ is an $\in$-isomorphism.

Notice that properties $(i),(i i)$ and (iii) above are formulas in the language of set theory. If the inner models $\mathcal{U} \cong \mathcal{U}^{\prime}$ are isomorphic, then the equivalence " $\mathcal{U} \models \sigma \Leftrightarrow \mathcal{U}^{\prime} \models \sigma$ " holds for every $L_{*}$-sentence $\sigma .{ }^{16}$ In the following, we denote by ${ }^{*} \mathrm{ZFC}_{o}$ the theory ${ }^{*} \mathrm{ZFC}$ without the axiom schema of saturation. The next theorem is proved in the Appendix.

\section{Theorem 3.1}

There exists an inner model with parameter $\mathcal{U}_{p}=\left\langle\mathbf{U}_{p}, \mathbf{E}_{p}, \otimes_{p}\right\rangle$ and a formula $\vartheta(x, y, p)$ such that $Z F C$ proves the following for every ultrafilter $D$ :

(i) $\mathcal{U}_{D}$ is a model of $* Z F C_{o}$.

(ii) $\Theta_{D}=\{\langle x, y\rangle: \vartheta(x, y, D)\}$ is an $\in$-isomorphism between the universe $\langle\mathbf{V}, \in\rangle$ and $\mathbf{W F}^{\mathcal{U}_{D}}$, the wellfounded part of $\mathcal{U}_{D} \cdot{ }^{17}$

(iii) If $D$ is countably incomplete $\kappa^{+}$-good, then $\mathcal{U}_{D} \models$ " $\Theta_{D}\left(\kappa^{+}\right)$-saturation

\footnotetext{
${ }^{15}$ Precisely, for every $a, b$, we say that $\mathcal{U} \models a \in b$ if $a, b \in \mathbf{U}$ and $a \mathbf{E} b ; \mathcal{U} \models *(a, b)$ if $a, b \in \mathbf{U}$ and $\otimes(a, b) ; \mathcal{U} \models \sigma \wedge \tau$ if $\mathcal{U} \models \sigma$ and $\mathcal{U} \models \tau$; etc. Notice that, for every formula $\sigma$ of $L_{*}$, " $\mathcal{U} \models \sigma\left(x_{1}, \ldots, x_{n}\right)$ " is a formula in the language of set theory.

${ }^{16}$ For more on the notion of inner (or syntactical) model, we refer the reader to [Ho] Ch. 5 .

${ }^{17} \mathrm{By}$ definition, $\mathbf{W} \mathbf{F}^{\mathcal{U}_{D}}$ is the $\in$-submodel of $\mathcal{U}_{D}$ whose universe is the class $\left\{u \in \mathbf{U}_{D}\right.$ : $\mathcal{U}_{D} \models$ " $u$ is wellfounded" $\}$.
} 
property". 18

As a direct consequence, we get

\section{Theorem 3.2}

For every model $M \models Z F C$ and for every $M$-cardinal $\kappa$, there is a model $N \models$ $Z F C_{o}$ such that $\mathbf{W F}^{N}=M$ and $N \models " \kappa^{+}$-saturation".

Proof. Take $D \in M$ with $M \models " D$ is a $\kappa^{+}$-good countably incomplete ultrafilter", and apply the previous theorem within $M$ with $D$ as a parameter. From the outside, we get a model $\left(\mathcal{U}_{D}\right)^{M}=\mathrm{ZFC}_{o}$ and an $\in$-isomorphism $\left(\Theta_{D}\right)^{M}: M \rightarrow\left(\mathbf{W F}^{\mathcal{U}_{D}}\right)^{M}$. By identifying each $m \in M$ with $\left(\Theta_{D}\right)^{M}(m)$, it is straightforwardly seen that $\left(\mathcal{U}_{D}\right)^{M}$ is the model $N$ for which we are looking.

For any model $M \models \mathrm{ZFC}^{-}$, denote by $\mathrm{Card}^{M}$ the set of its cardinals, and by $\operatorname{Def}^{M}$ the set of all $M$-definable cardinals. Precisely,

$$
\begin{aligned}
\operatorname{Card}^{M}=\{\kappa \in M: M \models & =\kappa \text { is a cardinal" }\} \\
\operatorname{Def}^{M}=\left\{\kappa \in \operatorname{Card}^{M}: M\right. & =\forall x\left(\varphi^{W F}(x) \leftrightarrow x=\kappa\right) \text { for some formula } \varphi(x) \text { with } \\
& \text { exactly one free variable }\}
\end{aligned}
$$

The above sets are linearly ordered by $\in^{M}$, the $M$-interpretation of the membership relation symbol. Notice that if $M=\mathbf{W F}^{N}$ is the wellfounded part of a model $N$, then $\operatorname{Card}^{M}=\operatorname{Card}^{N}$ and $\operatorname{Def}^{M}=\operatorname{Def}^{N}$. Recall the following definition for models of set theory. $M^{\prime}$ is an elementary end extension of $M$, in symbols $M \preceq_{e} M^{\prime}$, if $M \preceq M^{\prime}$ (i.e. $M$ is an elementary submodel of $M^{\prime}$ ) and for all $a \in M, M^{\prime} \models b \in a$ implies $b \in M$. Now assume that $M$ and $M^{\prime}$ are models of $\mathrm{ZFC}^{-}$. If $M \preceq M^{\prime}$ then $\operatorname{Def}^{M}=\operatorname{Def}^{M^{\prime}}$; and if $M \preceq_{e} M^{\prime}$ then $\left\langle\operatorname{Card}^{M}, \in^{M}\right\rangle$ is an initial segment of $\left\langle\operatorname{Card}^{M^{\prime}}, \in^{M^{\prime}}\right\rangle$, as linearly ordered sets. The next result shows that every model of ZFC can be extended to a model of ${ }^{*}$ ZFC. This gives a strong foundational justification to ${ }^{*} Z F C$.

\section{Theorem 3.3}

Every model $M=Z F C$ has an elementary end extension $M^{\prime}=\mathbf{W F}^{N}$ which is the wellfounded part of some model $N \models{ }^{*} Z F C$. Moreover, if Def $f^{M}$ is not cofinal in $\mathrm{Card}^{M}$, then one can take $M=M^{\prime}$.

Proof. If Def ${ }^{M}$ is cofinal in $\mathrm{Card}^{M}, \mathrm{Card}^{M}$ has countable cofinality and so, by a classic result of H.J. Keisler and M. Morley [KM], there exists a proper elementary end-extension $M^{\prime} \succeq_{e} M$. If $\operatorname{Def}^{M}$ is not cofinal in $\operatorname{Card}^{M}$, let $M^{\prime}=$ $M$. In both cases, it is possible to pick $\nu \in \operatorname{Card}^{M^{\prime}}$ such that $M^{\prime} \models " \nu>\kappa$ " for

\footnotetext{
${ }^{18}$ Definition and properties of good ultrafilters can be in found in $[\mathrm{CK}] \S 6.1$. In particular, recall that ultrapowers modulo countably incomplete $\kappa$-good ultrafilters are $\kappa$-saturated (provided the language has cardinality less than $\kappa$ ) and that $\kappa^{+}$-good countably incomplete ultrafilters exist for any infinite cardinal $\kappa$.
} 
all $\kappa \in \operatorname{Def}^{M}$. Now apply the previous theorem by considering the $M^{\prime}$-cardinal $\nu$ and get a model $N \models{ }^{*} \mathrm{ZFC}_{o}+$ " $\nu^{+}$-saturation", where $\mathbf{W F}{ }^{N}=M^{\prime} \succeq_{e} M$. Notice that $N$ satisfies the axiom schema of saturation. In fact, let $\kappa \in N$ be a "definable" cardinal, that is $\kappa \in \operatorname{Card}^{N}$ and $N \models \forall x \in \mathbf{S}\left(\varphi^{S}(x) \leftrightarrow x=\kappa\right)$. Then $\kappa \in \operatorname{Def}^{N}=\operatorname{Def}^{M^{\prime}}=\operatorname{Def}^{M}$, hence $N \models " \nu>\kappa "$ and $N=" \kappa$-saturation".

In particular, ${ }^{*} \mathrm{ZFC}$ is a conservative extension of $\mathrm{ZFC}$.

\section{Theorem 3.4}

ZFC is faithfully interpretable in * ZFC by relativizing quantifiers to the class of standard sets. That is, for every $\in$-sentence $\sigma$

$$
Z F C \vdash \sigma \Leftrightarrow{ }^{*} Z F C \vdash \sigma^{S}
$$

Proof. If ZFC $\forall \sigma$, then there is a model $M \models \mathrm{ZFC}+\neg \sigma$. Apply the previous theorem to find a model $N={ }^{*} \mathrm{ZFC}$ with $\mathbf{W F}^{N} \succeq_{e} M$. Notice that $M \models \neg \sigma \Leftrightarrow N \models(\neg \sigma)^{W F}$. Notice also that $(\neg \sigma)^{W F}$ is the same as $\neg(\sigma)^{S}$. Thus $N={ }^{*} \mathrm{ZFC}+\neg(\sigma)^{S}$ and this proves ${ }^{*} \mathrm{ZFC} \forall \sigma^{S}$. The reverse implication is similar. If $* \mathrm{ZFC} \forall \sigma^{S}$, then there is a model $N=* \mathrm{ZFC}+\neg\left(\sigma^{S}\right)$. In particular, $\mathbf{W F}^{N}=\mathrm{ZFC}+\neg \sigma$, hence $\mathrm{ZFC} \nvdash \sigma$.

\section{APPENDIX (Proof of Theorem 3.1).}

The following proof can be seen as the counterpart within ZFC of the construction of pseudo-superstructures given in [D1] in a nonwellfounded context. 19 Here, binary structures will be considered instead of transitive classes, and a "simulation" of the powerset operator will be used to construct a von Neumann hierarchy over an ultrapower of the universe. Since we deal with "nonstandard" membership relations over proper classes, some caution is needed in order to give a correct formalization of our proof. However, since all arguments involved should be in principle clear, we only give an outline of the construction of $\mathcal{U}_{D}$. Let $D$ be a given ultrafilter over a set $I$ and let $\sim$ denote the corresponding equivalence relation on the class of functions with domain $I$, that is $f \sim g \Leftrightarrow\{i \in I: f(i)=g(i)\} \in D$. Use Scott's trick to produce equivalence classes $[f]=\{g: g \sim f$ and $\forall h$ with $h \sim g, \rho(h) \geq \rho(g)\}$ which are sets rather than proper classes. Doing so, the universal $D$-ultrapower $\left\langle\mathbf{V}_{D}^{I}, \varepsilon\right\rangle$ can be defined as the structure whose universe is the class of all equivalence classes $[f]$, and where the membership relation is defined the usual way: $[f] \varepsilon[g] \Leftrightarrow$ $\{i \in I: f(i) \in g(i)\} \in D$. (Up to isomorphisms) $\mathbf{V}_{D}^{I}$ will be the class of internal sets of our nonstandard model $\mathcal{U}_{D}$, and the diagonal embedding

\footnotetext{
${ }^{19}$ Precisely, the framework adopted in [D1] is Zermelo-Fraenkel-Boffa set theory ZFBC, a variant of Zermelo-Fraenkel set theory where a well-ordering of the universe is available and a strong anti-foundation principle, namely Boffa's superuniversality [Bo], is postulated instead of regularity. ZFBC was first proposed for the foundations of nonstandard methods by D. Ballard and K. Hrbàček in [BH].
} 
$d: \mathbf{V} \rightarrow \mathbf{V}_{D}^{I}$ will be the nonstandard embedding. We now have to accomodate external sets so to get the whole model. To this end, we consider the von Neumann hierarchy. Precisely, start with $\mathcal{U}_{0}=\left\langle\mathbf{V}_{D}^{I}, \varepsilon\right\rangle$, and let $\chi_{00}$ be the identity map on $\mathcal{U}_{0}$. Proceeding by transfinite induction, assume that structures $\mathcal{U}_{\beta}=\left\langle\mathbf{U}_{\beta}, \varepsilon_{\beta}\right\rangle$ together with end extensions $\chi_{\beta^{\prime} \beta}: \mathcal{U}_{\beta^{\prime}} \rightarrow \mathcal{U}_{\beta}$ have been defined for all $\beta^{\prime} \leq \beta<\alpha$ in such a way that compositions $\chi_{\beta^{\prime} \beta} \circ \chi_{\beta^{\prime \prime} \beta^{\prime}}=\chi_{\beta^{\prime \prime} \beta}$ commute for all $\beta^{\prime \prime} \leq \beta^{\prime} \leq \beta<\alpha$ and $\chi_{\beta \beta}$ is the identity map on $\mathbf{U}_{\beta}$. Recall that an end extension $\chi:\langle A, E\rangle \rightarrow\langle B, F\rangle$ is a 1-1 $\in$-homomorphism where images pick no new elements, i.e. $b F \chi(a) \Leftrightarrow b=\chi\left(a^{\prime}\right)$ for some $a^{\prime} E a$. If $\alpha=\gamma+1$ is a successor, define $\mathbf{U}_{\gamma+1} \doteq\left\{x: x \subset \mathbf{U}_{\gamma}\right.$ is a set $\}$ and let $\chi_{\gamma, \gamma+1}: a \mapsto$ $\left\{a^{\prime} \in \mathbf{U}_{\gamma}: a^{\prime} \varepsilon_{\gamma} a\right\} \in \mathbf{U}_{\gamma+1}$. The membership relation on $\mathbf{U}_{\gamma+1}$ is defined as follows: $x \varepsilon_{\gamma+1} x^{\prime} \Leftrightarrow x=\chi_{\gamma, \gamma+1}(a)$ for some $a \in x^{\prime}$. As a consequence, $\chi_{\gamma, \gamma+1}: \mathcal{U}_{\gamma} \rightarrow \mathcal{U}_{\gamma+1}$ in an end extension. The remaining end extensions are then defined by composing $\chi_{\beta, \gamma+1}=\chi_{\gamma, \gamma+1} \circ \chi_{\beta, \gamma}$ for all $\beta<\gamma$. If $\alpha$ is limit, the structure $\mathcal{U}_{\alpha}$ and the end extensions $\chi_{\beta \alpha}$ for $\beta \leq \alpha$, are defined as the direct limit of the system $\left\{\mathcal{U}_{\beta} ; \chi_{\beta^{\prime} \beta}\right\}_{\beta^{\prime} \leq \beta<\alpha}$. Finally, let $\left\{\left\langle\mathbf{U}_{D}, \mathbf{E}_{D}\right\rangle ; \chi_{\alpha}\right\}_{\alpha \in O N}$ be the direct limit of the whole family $\mathcal{S}=\left\{\mathcal{U}_{\alpha} ; \chi_{\beta \alpha}\right\}_{\beta \leq \alpha \in O N}$. ${ }^{20}$ Notice that, in a way, the direct system $\mathcal{S}$ "simulates" an increasing $\mathbf{O N}$-sequence of transitive classes. Since all $\chi_{\beta \alpha}$ are end extensions, for any $x \in \mathbf{U}_{D}$, the collection $\left\{x^{\prime} \in \mathbf{U}_{D}: x^{\prime} \mathbf{E}_{D} x\right\}$ of its $\mathbf{E}_{D}$-elements is a set. Now, recall the following well-known property. "Suppose $\mathbf{T}$ is a transitive class such that for every set $x, x \subset \mathbf{T}$ implies $x \in \mathbf{T}$. Then $\langle\mathbf{T}, \in\rangle \models \mathrm{ZFC}^{-"}$. Similarly, it is proved that $\left\langle\mathbf{U}_{D}, \mathbf{E}_{D}\right\rangle \models \mathrm{ZFC}^{-}$as a consequence of the following fact. "If a set $x \subset \mathbf{U}_{D}$, then there exists $a \in \mathbf{U}_{D}$ such that $x=\left\{x^{\prime} \in \mathbf{U}_{D}: x^{\prime} \mathbf{E}_{D} a\right\} "$." By definition, each $\mathcal{U}_{\alpha}$ is obtained from $\mathcal{U}_{0}$ by iterating $\alpha$ times a "simulation" of the power set operator. As a consequence, a natural copy of $V_{\alpha}$ is enclosed in $\mathbf{U}_{\alpha}$, and an isomorphism $\Theta_{D}$ between the universal class $\mathbf{V}=\bigcup_{\alpha \in O N} V_{\alpha}$ and a subclass $\mathbf{V}^{\prime} \subset \mathbf{U}_{D}$ is defined. It is then straightforwardly checked that $\mathbf{V}^{\prime}$ is the wellfounded part of $\left\langle\mathbf{U}_{D}, \mathbf{E}_{D}\right\rangle$. The interpretation $\otimes_{D}$ of the nonstandard embedding is defined as the isomorphic copy of the diagonal embed$\operatorname{ding} d: \mathbf{V} \rightarrow \mathbf{V}_{D}^{I}=\mathcal{U}_{0}$ given by $\otimes=\chi_{0} \circ d \circ \Theta_{D}^{-1}$. The inner models $\mathcal{U}_{D}=\left\langle\mathbf{U}_{D}, \mathbf{E}_{D}, \otimes_{D}\right\rangle$ and the $\in$-isomorphisms $\Theta_{D}:\langle\mathbf{V}, \in\rangle \rightarrow \mathbf{W F}^{\mathcal{U}_{D}}$, satisfy the desired properties.

\section{References}

[BH] D. BAllard AND K. HRBÀC̆EK, Standard foundations for nonstandard analysis, J. Symb. Logic, vol. 57, 1992, pp. 741-748.

\footnotetext{
${ }^{20}$ According to the usual definition in category theory, a direct limit is unique only up to isomorphisms. We remark that, in order to correctly formalize our construction, we need to adopt a definition of direct limit which is uniquely determined. In fact, when proceeding by transfinite induction over all ordinals, one is not allowed to choose one object at each limit step.
} 
[Bo] M. Boffa, Forcing et négation de l'axiome de fondement, Mém. Acad. Sc. Belg., tome XL, fasc. 7, 1972.

[CK] C.C. Chang and H.J. Keisler, Model theory, 3rd edition, NorthHolland, 1990.

[D1] M. Di NAsso, Pseudo-superstructures as nonstandard universes, J. Symb. Logic, vol. 63, 1998, pp. 222-236.

[D2] M. Di NAsso, ${ }^{*} Z F C$ : an axiomatic *approach to nonstandard methods, C. R. Acad. Sc. Paris, t. 324, Série I, n. 9, 1997, pp. 963-967.

[D3] M. Di NAsso, On the foundations of nonstandard mathematics, Math. Japonica, vol. 50, 1999, pp. 131-160.

[D4] M. Di NASso, Elementary embeddings of the universe in a nonwellfounded context, in preparation.

[HL] A.E. HuRd AND P.A. Loeb, An introduction to nonstandard analysis, Academic Press, 1985.

[Ho] W. Hodges, Model theory, Cambridge University Press, 1993.

[HR] K. HrbÀC̆eK, Axiomatic foundations for nonstandard analysis, Fund. Math., vol. 98, 1978, pp. 1-19.

[JE] T. Jech, Set theory, Academic Press, 1978.

[KM] H.J. KeISLER AND M. MorLey, Elementary extensions of models of set theory, Israel J. Math., vol. 6, 1968, pp. 49-65.

[KU] K. KUnEn, Set theory: an introduction to independence proofs, North-Holland, 1980.

[RZ] A. Robinson AND E. ZAKOn, A set-theoretical characterization of enlargements, in Applications of model theory to algebra, analysis and probability, W.A.J. Luxemburg ed., Holt, Rinehart \& Winston, 1969, pp. 109-122.

Dipartimento di Matematica Applicata

Università di Pisa, via Bonanno 25/B

55126 Pisa, Italy

email: dinasso@dma.unipi.it 